\title{
Numerical Simulation of Shock Oscillations over Airfoil Using a Wall Law Approach
}

\author{
E. Goncalvès* and R. Houdeville ${ }^{\dagger}$ \\ ONERA, 31055 Toulouse CEDEX, France
}

\section{Introduction}

$\mathbf{S}$ HOCK-INDUCED oscillations (SIO) are a pure aerodynamic problem that can occur over rigid airfoils as a result of the development of instabilities caused by the boundary-layerseparation and the shock-wave interaction. This problem is extremely important because it can lead to the buffeting phenomenon through the mechanical response of the wing structure. A detailed description of the physical features of SIO is given by Lee. ${ }^{1}$ Computations have been essentielly done over thick airfoils to investigate the SIO problem. ${ }^{2-4}$

Numerically, the local time-step technique, which is efficient to accelerate the convergence towards the steady state, cannot be applied for unsteady computations that imperatively need a global time step. This constraint drastically reduces the method efficiency because of the Courant-Friedrichs-Lewy stability criterion. To overcome this difficulty, the dual time-stepping approach has been proposed by Jameson. It has also been used recently by Furlano et al. and Renaud et al. ${ }^{6}$ for SIO computations.

In the present study a wall law approach is used to relax the mesh refinement near the wall and therefore to increase the value of the global time step. Then, the computational efficiency of the explicit method is restored. Providing large CPU cost savings, the wall law approach has also proved to be attractive for the quality of its results in computing separated flows and for the robustness improvment it brings. $^{\text {? }}$

Moreover, the eddy viscosity models based on the linear Boussinesq relation are known to be unable to capture the boundarylayer separation and unable to take into consideration the nonequilibrium effects. A consequence of these weakness observed for unsteady computations is the overproduction of eddy viscosity, which limits the development of natural unsteadinessand modifies the flow topology. In this Note, we show that the turbulence model behavior can be remarkably improved by limiting the eddy viscosity with the shear-stress-transpott (SST) correction associated with a wall law approach.

\section{Numerical Methods}

A code solving the uncoupled Reynolds-averaged NavierStokes/turbulent systems for multidomain structured meshes is used for the present study. This code is based on a cell-centeredfinite volume discretization. Fluxes are computed with the Jameson scheme, and time integration is realized through a four-stage Runge-Kutta algorithm. More details about the solver can be found in Ref. 8 .

Various two-equation turbulence models are used in the present study: the Menter ${ }^{9}$ SST $k-\omega$ model and also the high-Reynoldsnumber version of the Jones-Launder ${ }^{10} k-\varepsilon$ model, with and without the Menter SST correction.

Presented as Paper 2001-2857 31st Fluid Dynamics Conference, Anaheim, CA, 11-14 June 2001; received 25 July 2001; revision received 10 August 2002; accepted for publication 9 April 2003. Copyright (C) 2003 by E. Goncalvès and R. Houdeville. Published by the American Institute of Aeronautics and Astronautics, Inc., with permission. Copies of this paper may be made for personal or internal use, on condition that the copier pay the $\$ 10.00$ per-copy fee to the Copyright Clearance Center, Inc., 222 Rosewood Drive, Danvers, MA 01923; include the code 0001-1452/03 \$10.00 in correspondence with the CCC.

* Assistant Professor, Aerodynamics and Energetics Modelling Department, 2, avenue Belin, B.P. 4025; Eric.Goncalves@ hmg.inpg.fr.

${ }^{\dagger}$ Research Master, Aerodynamics and Energetics Modelling Department, 2, avenue Belin, B.P. 4025; Robert.Houdeville@ onecert.fr. 
At the wall a no-slip condition is used coupled to a wall law treatment. It consists in imposing the diffusive flux densities, required for the integration process, in adjacent cells to a wall. More details concerning the wall law approach are given in Ref. 7. For unsteady boundary layers the existence of a wall law is assumed valid at each instant. As shown in Cousteix et al., ${ }^{11}$ the velocity phase shift is nearly constant in the logarithmic region and equal to the shift of the wall shear-stress phase. This is true for a Strouhal number up to 10 .

\section{Flow Conditions}

The experimentalstudy has been conductedin the S3MA ONERA wind tunnel ${ }^{12}$ with the supercritical RA16SC1 airfoil. The Mach number and the Reynolds number based on the chord are respectively $M_{\infty}=0.732$ and $R e_{c}=4.2 \times 10^{6}$. The angle of attack varies from 0 to $4.5 \mathrm{deg}$. Transition is fixed near the leading edge at $x / c=7.5 \%$ on both sides of the model.

For the computations experimental corrections are used in order to take into account wind-tunnel wall effects. The Mach number is decreased by 0.09 , and the angle of attack is decreased by $1 \mathrm{deg}$ at all incidences with respect to experiment. The grid has a C-type topology. It contains 321 nodes, 241 of which are on the airfoil. The $y^{+}$values of the coarse mesh, at the center of the first cell, vary between 1 and 80 for a steady computation at 4-deg angle of attack.

\section{Comparison to Experiments}

\section{A. Four-Deg-Incidence, $\boldsymbol{k}-\boldsymbol{\omega}$ SST Model}

To describe the buffet phenomenon, one period of the lift coefficient is divided into 10 equal parts, as shown in Fig. 1. The skin-friction coefficient $C_{f_{0}}=\tau_{w} / 0.5 \rho_{\infty} U_{\infty}^{2}$ is plotted in Figs. 2

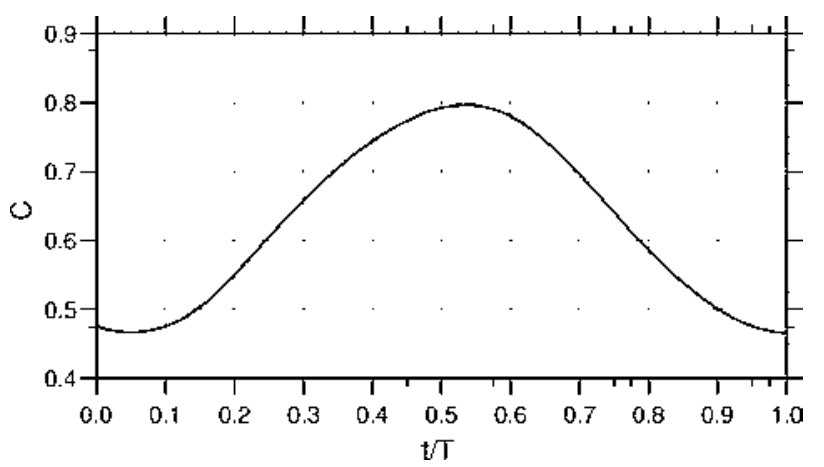

Fig. 1 Lift coefficient over one period of the cycle: 4-deg angle of attack, $k-\omega$ with SST correction.

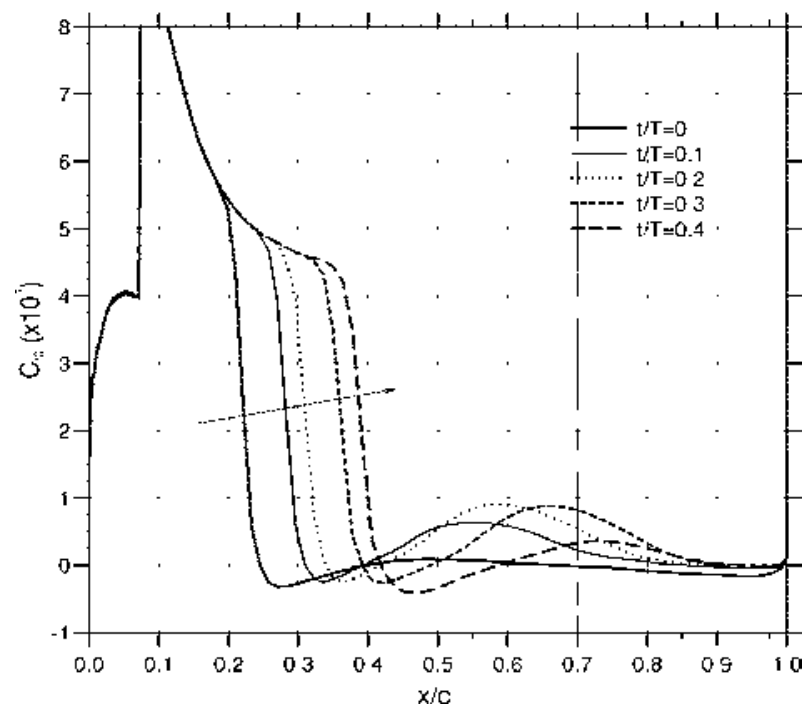

Fig. 2 Skin-friction coefficient during the downward shock displacement: 4-deg angle of attack, $k-\omega$ with SST correction.

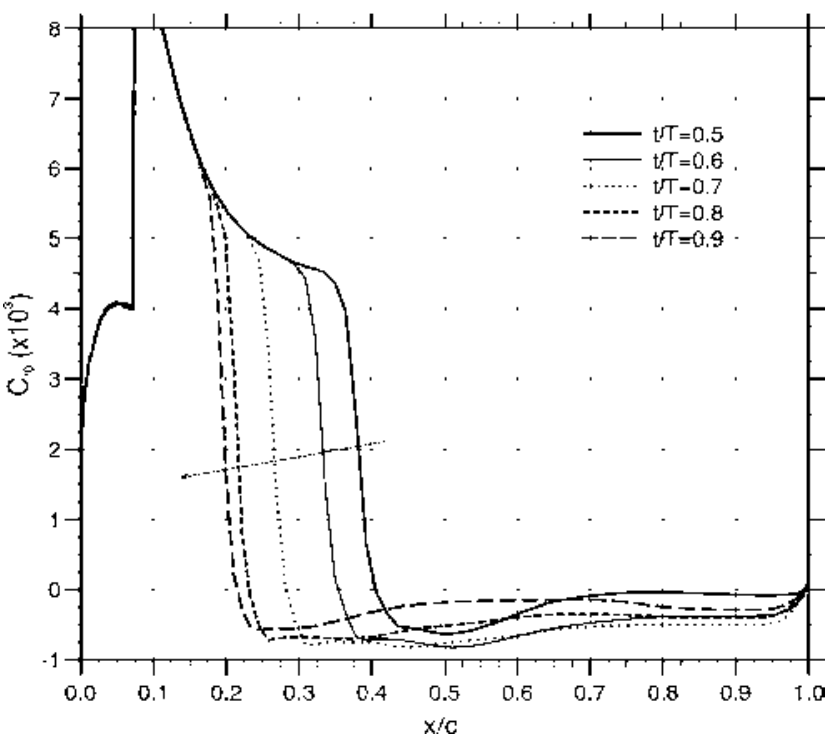

Fig. 3 Skin-friction coefficient during the upward shock displacement: 4-deg angle of attack, $k-\omega$ with SST correction.

and 3. At $t / T=0.5$ when the shock is at the most downstream position, separation occurs at $x / c=40 \%$ without reattachment before the trailing edge, although the backflow intensity is very small after $x / c=70 \%$. As the shock moves upward, the separated region increases and so does the backflow intensity, around $t / T=0.7$. The magnitude of the negative skin friction does not change a lot during the cycle near the shock. Actually, this change is important on the rear part of the airfoil. When the shock reaches its most upward position, reattachment takes place between $x / c=40$ and $70 \%$, and the strength of the backflow is strongly reduced. With the downward shock displacement the intensity of the skin friction increases in the reattached region, up to $x / c=90 \%$. A small separation bubble is always present near the foot of the shock.

The development of a mixing layer downstream of the shock can be evidenced by using the Q criterion. During the downward shock displacement, the vortices propagate, with pairing, up to the trailing edge. As the shock moves upward, they are shed in the wake and dissipated very quickly (less than one chord from the trailing edge).

\section{B. Other Results}

The rms values of the pressure fluctuations are compared in Fig. 4 with experimental results for three turbulence models and 4-deg angle of attack. The pressure side is represented by the negative values of the abscissa $x / c$. The maximum value is obtained at $x / c=40 \%$, just after the most downward shock location. The largest discrepancy with experiment is observed downstream the shock location, on the rear part of the airfoil and at the trailing edge.

Figure 4 clearly shows the influence of the SST correction. With the baseline Menter $k-\omega$ model no unsteady fluctuations develop at $\alpha=4 \mathrm{deg}$. With the $k-\varepsilon$ model shock-induced oscillations exist, but the amplitude of the pressure fluctuations is underestimated. The SST correction increases the oscillation amplitude up to the correct level over all of the suction side. Unfortunately, it also overestimates the pressure fluctuation on the pressure side.

The use of the SST correction and the use of a wall law approach, which removes the wall damping functions in separated regions, are the key points of the present study, both for the numerical efficiency of the time integration and for the quality of the results.

The reduced frequency $2 \pi f c / U_{\infty}$ of the SIO phenomenon and the rms amplitude of the lift coefficient are plotted in Figs. 5 and 6 as a function of the angle of attack. The experiment clearly shows that the reduced frequency increases with incidence. This tendency is reproduced by the computations and particularly well with the $k-\omega$ SST model, which gives the reduced frequency within $10 \%$ of the experimental results. The $k-\varepsilon$ model with SST correction does not seem to render this evolution as well. However, this model correctly 


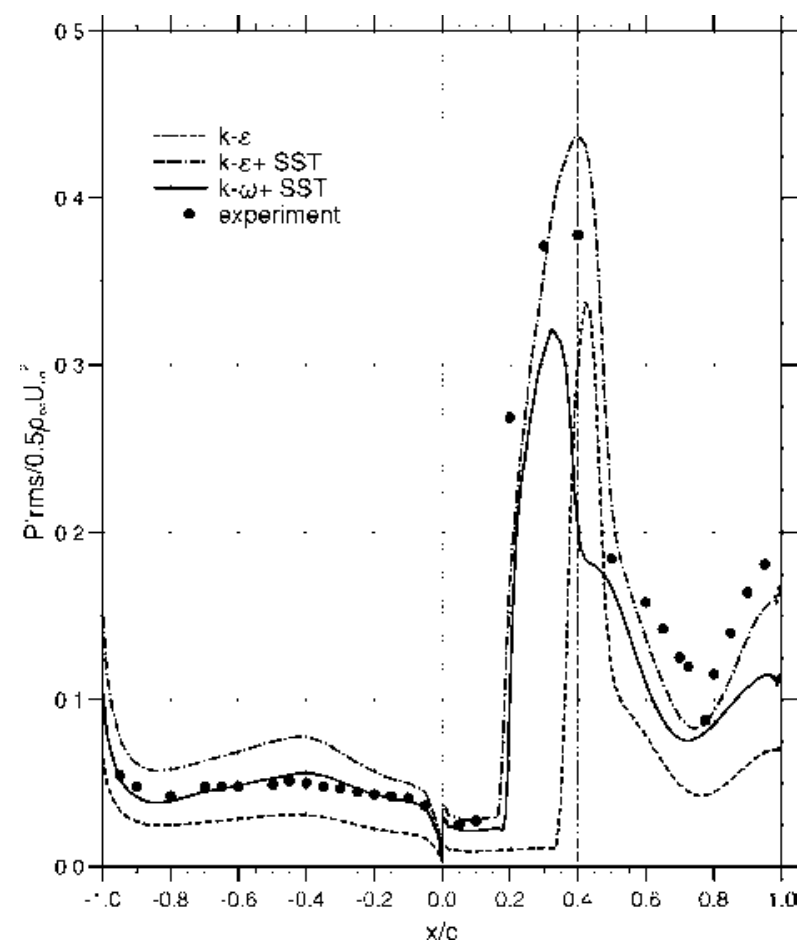

Fig. 4 Rms pressure fluctuations over the airfoil: 4-deg angle of attack, $k-\omega$ and $k-\varepsilon$ models.

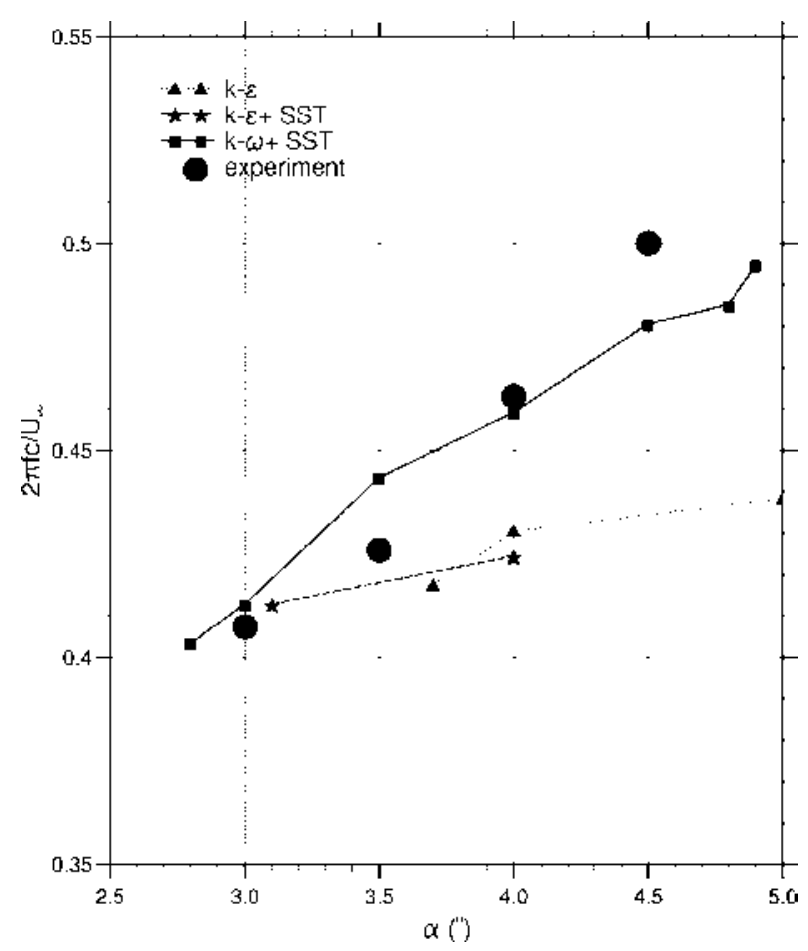

Fig. 5 Reduced frequency parameter vs angle of attack.

predicts the entrance in the SIO domain. Without the SST correction the corresponding angle of attack is shifted by $0.6 \mathrm{deg}$.

The amplitude of oscillations of the lift coefficient is probably more difficult to compute than the reduced frequency parameter because it directly relies on the correct description of the separated region. The experiment shows that the SIO phenomenon occurs just before the 3-deg angle of attack. The amplitude reaches a maximum value at 4 -deg incidence and decreases at larger values. This evolution is remarkably well reproduced by the $k-\omega$ SST model, which indicates a return to a steady state at 5-deg incidence. Unfortunately, there are no experimental results at this incidence because

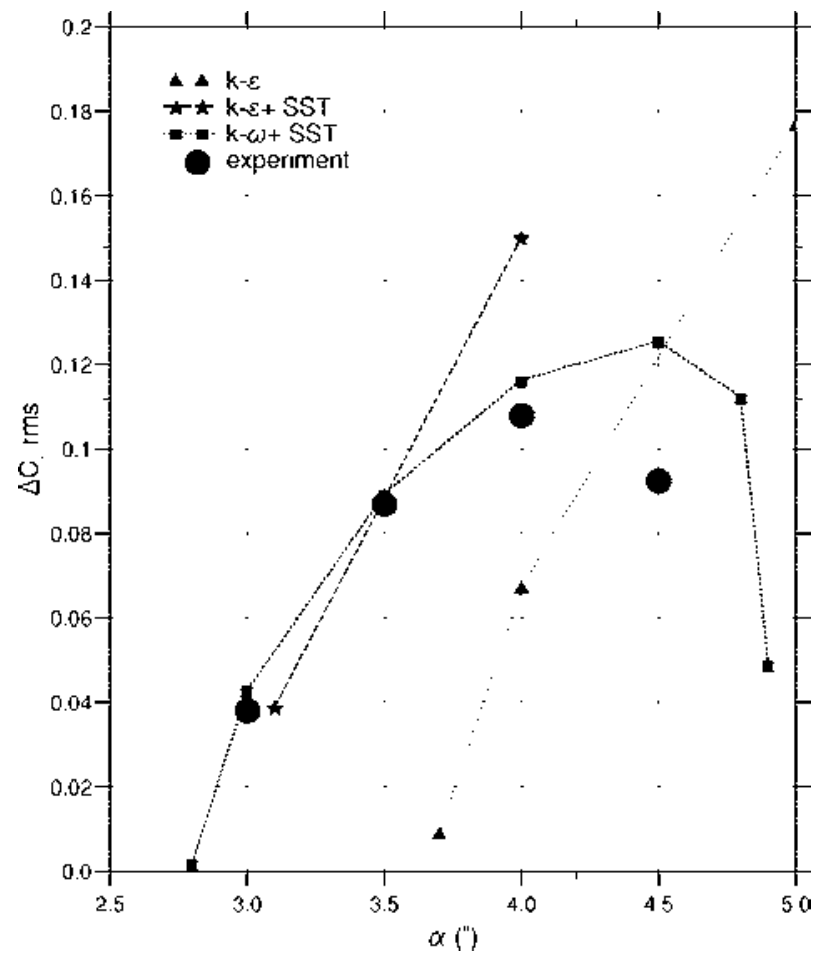

Fig. 6 Amplitude of the lift coefficient fluctuation vs angle of attack.

the emphasis has been put on the buffet appearance. The $k-\varepsilon$ model with SST correction also correctly predicts the entrance in the SIO domain but overpredicts the amplitude of oscillations at 4-deg incidence. Without the SST correction the results are shifted by 0.6-deg incidence, as for the reduced frequency parameter.

\section{Conclusions}

The prediction of shock-inducedoscillationshas proved to be sensitive to the turbulence modeling and numerical integration methods. The present Note shows the interest of the use of a wall law approach for unsteady computations over a transonic airfoil. First, the numerical efficiency and the CPU cost saving by relaxing the mesh are very significant. Moreover, the treatment associated to the $k-\omega$ and $k-\varepsilon$ models with the SST limiter is able to predict periodic self-sustained oscillations for the RA16SC1 airfoil correctly. Not only is the frequency of the SIO correctly computed, but also its evolution with the angle of attack as well as the amplitude of the lift coefficient. Comparisons with experimental values of the rms pressure fluctuations clearly indicate the great influence of the SST correction for the quality of results.

\section{References}

${ }^{1}$ Lee, B., "Self-Sustained Shock Oscillations on Airfoils at transonic speeds," Progress in Aerospace Sciences, Vol. 37, 2001, pp. 147-196.

${ }^{2}$ Raghunathan, S., Gillan, M., Cooper, R., Mitchell, R., and Cole, J., "Shock Oscillations on Biconvex Aerofoils," Aerospace Science and Technology, Vol. 3, 1999, pp. 1-9.

${ }^{3}$ Barakos, G., and Drikakis, D., "Numerical Simulation of Transonic Buffet Flows Using Various Turbulence Closures," InternationalJournal of Heat and Fluid Flow, Vol. 21, 2000, pp. 620-626.

${ }^{4}$ Wang, D., Wallin, S., Berggren, M., and Eliasson, P., "A Computational Study of Unsteady Turbulent Buffet Aerodynamics," AIAA Paper 20002657, June 2000.

${ }^{5}$ Furlano, F., Goncalves, E., Houdeville, R., and Coustols, E., "Unsteady RANS Computations: Simulation of the Buffeting over an Airfoil," IUTAM Symposium on Unsteady Separated Flows, International Union of Theoretical and Applied Mechanics, Toulouse, France, April 2002.

${ }^{6}$ Renaud, T., Corre, C., and Lerat, A., "Efficient Numerical Simulation of Buffet for Airfoils in Transonic Regime," IFASD, Madrid, June 2001.

${ }^{7}$ Goncalves, E., and Houdeville, R., "Reassessment of the Wall Functions Approach for RANS Computations," Aerospace Science and Technology, Vol. 5, 2001, pp. 1-14. 
${ }^{8}$ Couaillier, V., "Numerical Simulation of Separated Turbulent Flows Based on the Solution of RANS/Low Reynolds Two-Equation Model," AIAA Paper 99-0154, Jan. 1999.

${ }^{9}$ Menter, F., "Two-Equation Eddy-Viscosity Turbulence Models for Engineering Applications," AIAA Journal, Vol. 32, No. 8, 1994, pp. $1598-1605$.

${ }^{10}$ Jones, W., and Launder, B., "The Prediction of Laminarization with a Two-Equation Model of Turbulence," International Journal of Heat and Mass Transfer, Vol. 15, 1972,pp. 301-314.

${ }^{11}$ Cousteix, J., Houdeville, R., and Javelle, J., "Response of a Turbulent Boundary Layer to a Pulsation of the External Flow with and Without Adverse Pressure Gradient," IUTAM Symposium, International Union of Theoretical and Applied Mechanics, Toulouse, France, May 1981.

${ }^{12}$ Benoit, B., and Legrain, I., "Buffeting Prediction for Transport Aircraft Applications Based on Unsteady Pressure Measurements," AIAA Paper 87 2356, Aug. 1987. 